\title{
SIMULATION ASSESSMENT OF NEW GENERATION NAVIGATION STRATEGIES
}

\author{
Lídia Montero \\ M. Paz Linares \\ Department of Statistics and Operations Research \\ and inLab FIB \\ Universitat Politècnica de Catalunya-UPC \\ Carrer Jordi Girona 1-3 \\ 08034-Barcelona, SPAIN
}

\author{
Josep Casanovas-Garcia \\ Barcelona Supercomputing Center, \\ Department of Statistics and Operations Research \\ and inLab FIB \\ Universitat Politècnica de Catalunya-UPC \\ Carrer Jordi Girona 1-3 \\ 08034-Barcelona, SPAIN
}

\author{
Gonzalo Recio \\ Ester Lorente \\ Juan Salmerón \\ inLab FIB \\ Universitat Politècnica de Catalunya-UPC \\ Carrer Jordi Girona 1-3 \\ 08034-Barcelona, SPAIN
}

\begin{abstract}
Probe vehicle (or connected car) data is becoming an important source of real-time travel information for a variety of intelligent transportation system applications. Since traditional sensors have significant installation and maintenance costs, technological companies are interested in traffic data from these alternative detection techniques for computing traffic aware shortest routes. This paper analyzes and evaluates the use of data provided by probe vehicles in two reactive navigation strategies and how this affects a set of city and driver key performance indicators. The case study adopts a microscopic simulation approach to emulate real-size fleets of probe cars providing positions and speed data. The paper presents and discusses the modeling approach and the obtained results after conducting an experimental design for a Barcelona district scenario. Moreover, a simulation-based framework is introduced for simplifying simulation results analysis and easily visualizing origin-destination paths for the proposed driver segments (experts, regular and tourists).
\end{abstract}

\section{INTRODUCTION}

In urban areas, connected vehicles are expected to reach high concentrations in the immediate future. The market of connected vehicles is booming and both academia and the automotive industry are promptly respondering to its requirements. In fact, there is a broad consensus on the new family of services that will be enabled by the advances in vehicle-to-vehicle communication.

The use of probe vehicle data (PVD) has been investigated in some research projects such as Mobile Millenium or CarTel (Hull et al. 2006), which included a pilot traffic-monitoring system using the GPS in cellular phones to gather traffic information, process it, and distribute it back in real time to the phones. Products and companies performing mobile crowdsourcing (Google Traffic, INRIX and TomTom Traffic) allow for real-time data gathering. 
The capabilities to effectively monitor traffic conditions have been studied from early considerations of equipped vehicles as a mobile sensors network (Hull et al. 2006) to more recent surveys (Lee and Gerla 2010; Bessler and Paulin 2013). However, most analyses have been conducted from the perspectives of either continuous monitoring of the vehicle state or driver safety, while some others have considered the potential guidance offered by such mobile sensing (Lorkowski et al. 2003; Lee and Park 2008; Wang et al. 2017). This last area of analysis is the scope of the current paper.

Without trying to be exhaustive, the impact of route guidance on travel time, environment and safety has been investigated (Oh and Jayakrishnan 2002; Paikari et al. 2013; Olia et al. 2016), usually in relation to its benefit under incident conditions and while quantitatively assessing the potential impacts of realtime routing guidance and advisory warning messages to guided vehicles. Some other authors have analyzed reactive policies (Deflorio 2003) using simulation and proactive route guidance (Pan et al. 2012). Nevertheless, in some of these studies, the elaboration of traffic state estimation has been treated from a simplistic point of view or has not been described in depth. For example, (Pan et al. 2012) use Greenshield's model to estimate travel times in road segments, but this approach does not seem suitable for urban networks and details are not included.

The aim of this paper is to present an experimental design for simulation assessment of navigation strategies based on PVD while considering driver behavior and route choice models. Moreover, a simulation-based framework is introduced for simplifying simulation results analysis and easily visualizing them. The conceptual framework is briefly described first, and a section follows describing in detail the emulation of the different concepts included in the Custom Module. After that, we describe the simulation experiments for a large fleet of PVDs according to penetration rates and additional factors considered in the experimental design. The next section provides an analysis of the results and the paper ends with our conclusions and future research.

\section{CITSCALE: A SIMULATION TESTBED FRAMEWORK}

The CitScale tool (Linares et al. 2017) is a software platform developed by inLab FIB at Universitat Politècnica de Catalunya (UPC) for visualizing, analyzing and comparing experimental designs that model urban traffic scenarios. Assessments of new mobility concepts and new automotive vehicles are the main targets of projects that use CitScale (Montero et al. 2017). In particular, in this paper, this tool offers the possibility of enabling a new family of services based on the previously mentioned advances in inter-vehicular communications. In particular, the proposed case study aims at evaluating the effect of using probe car fleet data at different penetration levels to develop real-time navigation strategies for connected vehicles.

Figure 1 shows the proposed simulation testbed framework that is composed of an Executions Controller, a Traffic Simulation Module, a Result Preparation Module and a Visual Analytics Module. The core components of the CitScale analytics platform are the graphical interface and the traffic simulation model.The traffic simulation component includes a microscopic traffic simulator and a set of custom modules and functions that were developed using API extensions.

The analysis of simulation results is performed by two fundamental and independent components: the Result Processing Module and the Visual Analytics Module. One deals with the automated preparation of data and the other with operating the data display. The Result Processing Module makes the entire preprocessing of data automatically, thus allowing the Visual Analytics Module to automatically use the simulation results directly without the need for any manual update.

The visualization and analysis tool has been implemented using the Shiny (RStudio Project) web application for $\mathrm{R}$ that simplifies the development of interactive web applications. The Shiny web application is agnostic to the traffic simulation platform. If we are going to emulate smart city policies by necessarily evaluating new vehicle types, probe vehicle sensors, navigation strategies and innovative mobility concepts such as multiple passenger ridesharing, then several ad hoc components in the traffic 


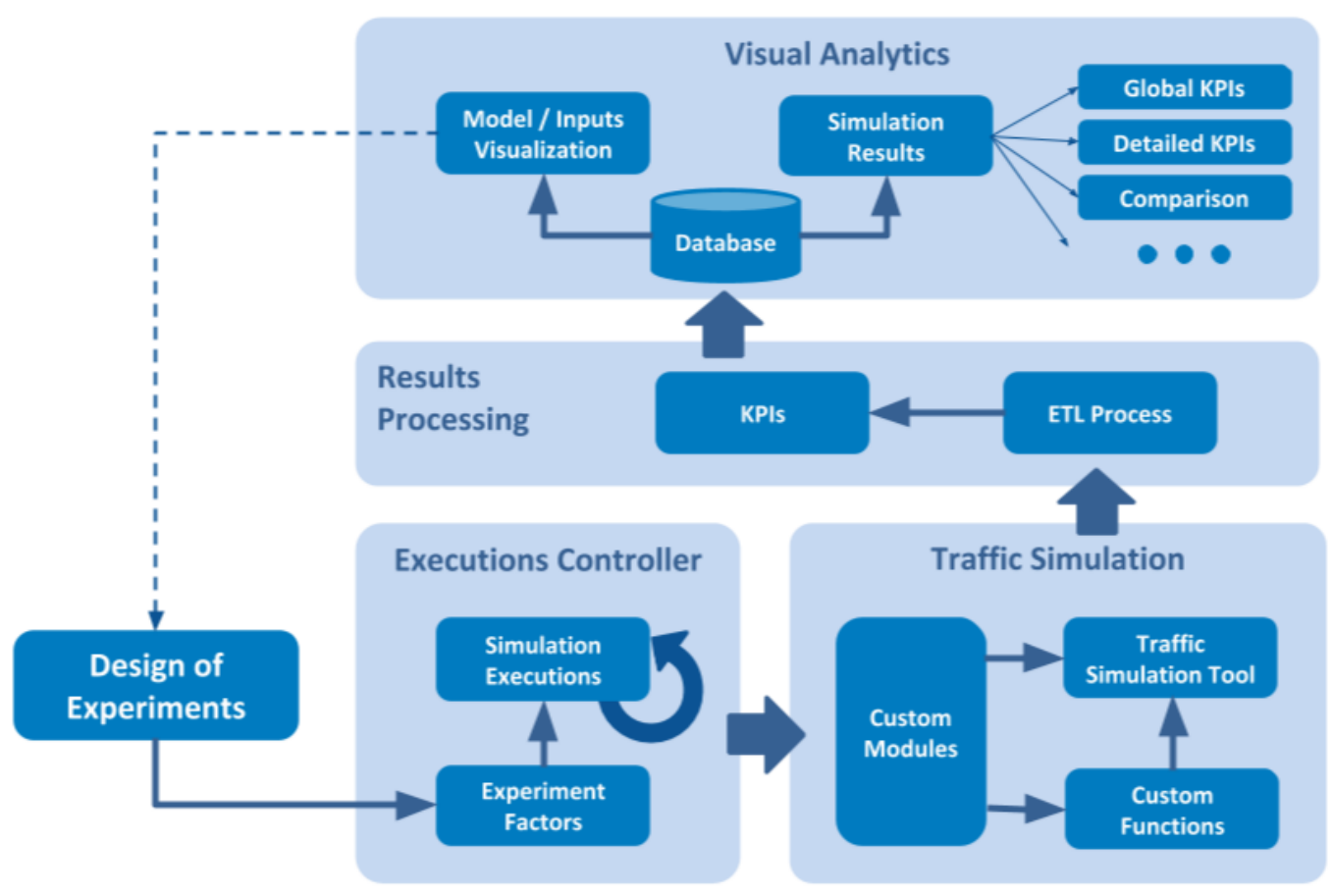

Figure 1. Simulation Testbed Architecture.

simulation model must be programmed using API extensions. However, any microsimulation platform that allows API extensions can be used.

In this work, an Aimsun (Transport Simulation Systems 2014) model was available from previous projects. Aimsun functional architecture and the interaction libraries support the extended modelingutilities that are required. The exchange of information between the API applications and the microsimulator can be made at every simulation step $(0.5 \mathrm{sec})$. The programming languages in which Aimsun provides its API are $\mathrm{C}++$ and Python. While Python is used to easily collect some of the data, $\mathrm{C}++$ is needed for emulating the probe vehicles due to performance reasons.

\section{CUSTOM MODULES IMPLEMENTATION}

The proposed case study requires detailed driver behavior, heuristic route choice modeling that depends on knowledge of the network and congestion, and the availability of navigation data. Traffic microsimulation capabilities are needed to achieve the final goal.

In order to extend the standard functionalities of the selected microsimulation tool so that it can deal with the requirements of this case study, the next items have been implemented into the Custom Modules: PVD emulation, driver behavior modeling, navigation strategies and time-dependent link and lane travel time estimation module.

\subsection{Probe Vehicle Data Emulation}

The objective of this module is to emulate vehicles with equipped sensors connected to a Traffic Management Center. This paper assumes that the Vehicle-to-infrastructure (V2I) and Vehicle-to-vehicle (V2V) technology is on board probe cars (Montero et al. 2016). The collected data were filtered to remove any incomplete observations or outliers. Then, this data was used to calibrate the emulation of the PVD module included in the Traffic Simulation Module. 


\section{Montero, Linares, Casanovas-Garcia, Recio, Lorente, Salmerón}

The main aim of this paper is to emulate probe car 'real-time data' use in connected car guidance under different levels of probe vehicle penetration. To this end, only basic vehicle sensors have been assumed, allowing at each simulation step data for vehicle position and speed.

\subsection{Driver Behavior Modeling}

This paper highlights driver behavior modeling, which has not been considered in any related papers in the literature (Lee and Park 2008; Wang et al. 2017; Paikari et al. 2013; Olia et al. 2016; Deflorio 2003; Pan et al. 2012; Minelli et al. 2015). Drivers are split into six groups according to their knowledge of network and traffic conditions and according to guidance availability:

- Expert drivers: those who know the network and historic traffic conditions for the selected horizon of study. They are modeled with route choice selection and proportions following experienced travel times that satisfy dynamic user equilibrium (DUE) (Chiu et al. 2011) by assuming a historic demand pattern. DUE paths and proportions are loaded in the simulation environment from a pre-calculated binary file.

- Regular drivers: those with knowledge of the network and historic traffic conditions for recurrent trips (50\% randomly selected), but who use main streets based on free-flow for non-recurrent trips $(50 \%)$.

- Tourist drivers: who have limited knowledge of network and traffic conditions and use K-shortest paths algorithm based on the main streets.

Finally, Guided drivers constitute a design-dependent proportion for any Expert, Regular or Tourist driver class, and they are modeled with a $100 \%$ acceptance of navigation advice.

Expert and Regular drivers exhibit driving characteristics related to the car-following model, such as reaction times, desired speed and acceptance of speed limitations. According to the calibrated profile of Barcelona drivers: Reaction Time (1.0s); Reaction Time at Stop (1.35s); Reaction Time at Traffic Light (1.35s); Speed Acceptance and Minimum Inter-Vehicular Distance are assumed to be truncated normally distributed with the former having mean-1.1, sd-0.1, min-0.9 and max-1.3, and the latter having mean$1.0 \mathrm{~m}, \mathrm{sd}-0.3 \mathrm{~m}$, min- $0.5 \mathrm{~m}$ and $\mathrm{max}-1.5 \mathrm{~m}$.

Tourist drivers behave roughly with a $25 \%$ increment in reaction times, means and limits (same standard deviation), and they strictly adhere to the speed limits, while Minimum Inter-vehicular Distance is truncated normally distributed with mean- $1.25 \mathrm{~m}$ and sd- $0.1 \mathrm{~m}$ - between 0.75 and $1.5 \mathrm{~m}$.

\subsection{Navigation Strategies}

An interesting discussion about route guidance assessment can be found at Wang et al. (2017). A navigation application is modeled as being available to a certain percentage in each driver classes. Three navigation strategies are implemented according to the simulation platform possibilities:

- Free-flow K-shortest paths when probe vehicle fleet is not available.

- Stochastic Route Choice considering instantaneous traffic conditions inferred from data provided by PVD. They are modeled from estimated instantaneous K-shortest travel time paths. They can be either link-based, according to user-defined link costs (link travel time estimates from PVD), or lane-based, according to user-defined stream costs (lane travel time estimates combined into a stream travel time).

To consider dynamic and instantaneous travel time based route choice, $100 \%$ re-routing is enabled every time window interval.

The critical point is that a real-time routing strategy for connected cars (guided) is applied from the travel times provided by the floating car data. Travel time estimates used in K-shortest path calculations 
might depend either on lane-based or overall link-based travel time, both of which rely on PVD sent to a centralized system.

Travel times between point of interest can be inferred according to Origin-Destination (OD) path travel times and route-choice proportions to feed Kalman filtering formulations proposed by the authors to estimate dynamic OD matrices.

\subsection{Link and Lane Travel Time Estimation Module}

In order to estimate time-dependent lane and link travel times, a proposal based on (Sanaullah et al. 2013) has been considered. This approach involves Time-window concept, which is an experiment design factor that represents the time interval in which travel times are being updated. Therefore, with a greater timewindow, the probability of finding lanes and links with available data grows.

The simulator can gather probe vehicle data (such as position and instant speed) at every simulation step (0.5 seconds). For our experiments, the time-window parameter choice is 2 seconds, which means that probe vehicle data is being collected periodically after this interval of time. This assumed timewindow is adequate in order to ease computational and memory costs.

The implemented approach for estimating travel time considers three different cases for every lane.

a) Case in which there are no PVD in the last time window.

b) Case in which there is PVD from just one car in the last time window.

c) Case in which there is PVD from more than one vehicle in the latest time-window.

In case (a), travel time estimation is the same as the lane travel time of the most recent time window. If there is no data available, the lane travel time is set to the time corresponding to a free flow situation.

In case (b), the following method is applied:

- Let $t t_{1}$ be the estimated travel time for the fraction of the lane until the first detection of the vehicle. Therefore, $t t_{1}$ is the length of this lane fraction divided into the vehicle's instantaneous speed at the first observation in this lane. Intuitively, it is the travelled distance on the lane, which gives a time magnitude.

- Let $t t_{L}$ be the difference (in seconds) between the first and last detection intervals.

- Let $t t_{2}$ be the estimated travel time for the last fraction of the lane since the last detection of the vehicle. Similarly as $t t_{1}, t t_{2}$ is computed as the distance from the last observation at the beginning of the next section divided by the probe vehicle's instantaneous speed in the last observation (detection interval) of the time-window.

Then, the travel-time of the considered lane is $t t_{1}+t t_{L}+t t_{2}$.

In case (c), a weighted travel time of the lane is computed considering all vehicles. Supposing there are $n$ cars providing data in the lane, $\frac{1}{n} \sum_{v=1}^{n} w_{v} t t_{v}$, where $t t_{v}$ is the travel time of a car calculated as explained in case $\mathrm{b}$ ), and the weight $w_{v} \in[0,1]$ is the fraction of the section length where prove vehicle $v$ is detected in the lane.

On the other side, link travel time estimation from PVD is computed as the mean of travel times for streams. And lane travel time estimation is performed combining stream travel times with the information about the allowed turns.

\section{DESIGN OF EXPERIMENTS}

The selected scenario is the Barcelona Central Business District, known as "L'Eixample" (Montero et al. 2016), which comprises $7.46 \mathrm{~km} 2$ and 250,000 inhabitants. The horizon study is $1 \mathrm{~h}$, accounting for 42,500 trips. Passenger car demand is modeled as 15-min time-sliced demand whose OD pattern reproduces the $9-10 \mathrm{~h}$ morning period in L'Eixample. 


\subsection{Design Factors}

The factors considered in the design of the simulation experiments (see Table 1) are:

- Driver Type Distribution (TD factor) into Expert-Regular-Tourist.

- Guidance Penetration (GP factor). Connected cars percentage of cars whose route choice decisions follow those advised by a Navigation Tool fed by PVD.

- Demand Pattern (DP factor) into 4 levels referring to a perturbation of the historic demand pattern in OD pairs belonging to the fourth percentile trip distance (according to Manhattan distance).They account for 42,500, 44,600, 46,860 and 48,600 trips, respectively. $0 \%$ means historic demand pattern

- Probe Vehicle Penetration percentage (PVD factor) modeled common to any driver type into 8 levels. Base level is 0\%. It indicates route guidance based on free-flow travel times. An additional Ground Truth level consisting on travel time estimates directly from 'simulated Ground Truth' was also included in some initial experimentation.

- Navigation Strategy (NS factor) modeling driving recommendations based on either lane-level or link-level PVD when PVD is available. Base level is lane-level, when 0\% PVD is set, free flow travel times are assumed.

- Time-Window length (TW factor) is the rolling horizon interval considered for the estimation of traffic variables from PVD. TW is not affected when 0\% PVD is set.

Table 1: Design factors description.

\begin{tabular}{rcll}
\hline Design Factors & \multicolumn{1}{c}{ Factor Levels } & Base Level & \multicolumn{1}{c}{ Alternative Levels } \\
\hline Driver Type Distribution & expert-regular-tourist & $40-50-10(\%)$ & $20-70-10,40-40-20$, \\
& & & $60-20-20(\%)$ \\
Guidance Penetration & guided-nonguided & $0-100(\%)$ & $10-90,20-80,30-70,50-50,70-$ \\
& & & $30,80-20,90-10,100-0(\%)$ \\
Demand Pattern & & $0(\%)$ & $10,20,30(\%)$ \\
Probe Vehicle Penetration & & $0(\%)$ & $10,20,30,80,90,100(\%)$ and \\
& & 3 min & PI (perfect info) \\
Time-window Length & & & 1.5 min, 6min \\
\hline
\end{tabular}

\subsection{Collected Key Performance Indicators}

In general, on microscopic traffic simulation platforms the default statistics are very rich. Statistics have been collected every 90s and stored in an SQLITE database for each replication. Driver Key Performance Indicators (KPIs) collected for each Expert, Regular and Tourist driver type (either guided or nonGuided) are shown in Table 2.

Table 2: Driver KPIs.

\begin{tabular}{rc}
\hline KPI & Unit \\
\hline Average Delay while covering $1 \mathrm{~km}$ & $\mathrm{~s} / \mathrm{km}$ \\
Average Speed per vehicle & $\mathrm{km} / \mathrm{h}$ \\
Average Travel Distance per vehicle & $\mathrm{Km}$ \\
Average Travel Time per vehicle & $\mathrm{Min}$ \\
Average Travel time to cover $1 \mathrm{~km}$ & $\mathrm{~s} / \mathrm{km}$
\end{tabular}

Network KPIs (see Table 3) are global statistics (all driver classes) and, over the whole simulation horizon. 
Montero, Linares, Casanovas-Garcia, Recio, Lorente, Salmerón

Table 3: Network KPIs.

\begin{tabular}{|c|c|c|}
\hline KPI & unit & ACRONYM \\
\hline Average Flow & $\mathrm{veh} / \mathrm{h}$ & mflow \\
\hline Average Speed & $\mathrm{km} / \mathrm{h}$ & mspeed \\
\hline Average Travel Time to cover $1 \mathrm{~km}$ & $\mathrm{~s} / \mathrm{km}$ & mtt.s.km \\
\hline Density & $\mathrm{veh} / \mathrm{km}$ & density \\
\hline Fuel Consumption & 1 & fuelc \\
\hline Mean Delay Time while covering $1 \mathrm{~km}$ & $\mathrm{~s} / \mathrm{km}$ & mdelay.s.km \\
\hline Throughput Rate (completed trips/total demand) & $\%$ & thrputrate \\
\hline Total CO2 emissions & $\mathrm{kg}$ & $\mathrm{CO} 2$ \\
\hline Total NOX emissions & $\mathrm{kg}$ & NOx \\
\hline Total Travel Distance & $\mathrm{km}$ & ttdis \\
\hline Total Travel Time & $\mathrm{h}$ & ttt.h \\
\hline Number of vehicles lost in system. & vehs & virlostin \\
\hline Number of vehicles lost out system. & vehs & virlostout \\
\hline Number of vehicles waiting to enter into the system. & Vehs & virwait \\
\hline Waiting Rate $=$ VirWait $/$ Demand & $\%$ & waitrate \\
\hline Number of vehicles inside the network & vehs & tvehin \\
\hline Total number of vehicles that have entered the system & vehs & inputveh \\
\hline Demand $=$ inputveh + virWait & vehs & demand \\
\hline Total number vehicles that have exited the system & vehs & tvehout \\
\hline
\end{tabular}

From a driver satisfaction point of view, the critical KPI is considered to be the average travel time (min), but also for researchers interested in the assessment of travel times between POIs (Points of Interest) inferred from PVD. A detailed analysis on the base scenario for all design factors found that five replications facilitates a global 5\% relative precision in average travel time at $95 \%$ confidence for any driver type, while the greatest absolute error was about $1 / 3$ min for tourist drive type.

For computational reason, running the full factorial design is unfeasible since $3,072 \times 5=15,360$ replications would be needed. Therefore, the first set of experiments was constrained in order to identify non-aliased factor main effects according to the Fedorov algorithm (Fedorov 1972) for optimal designs: 29 experiments were given (thus, 145 replications were executed, each one taking around $2 \mathrm{~h}$ in an Intel Core i7-4790 CPU (frequency of 3.6GHz)-4 cores-8GB DDR3 Memory and Windows 8.1 (x64 system)).

\section{SIMULATION RESULTS ANALYSIS}

Traffic simulation platforms provide a wide extend of KPI results. In this work, we have selected a few KPIs that were considered relevant for the evaluation of navigation systems. Nevertheless KPIs cannot be considered isolated, because they are strongly correlated. Figure 2 on the left shows Spearman coefficient of correlation (non-parametric statistic for the linear association between non-Gaussian numeric variables) between selected KPIs. It can be seen that most of the cells show an absolute value of Spearman correlation greater than 0.5 , so a monotonic relationship appears among most KPI pairs. For example, demand (total demand trips, trips entering plus waiting to enter in the system) and mtt.s.km (travel time to cover one $\mathrm{km}$ ) show a positive Spearman correlation of 0.77 indicating a direct relation: as total trips increases, travel time per $\mathrm{km}$ also increases, because traffic congestion gets worse. A normalized principal component analysis (PCA) has been addressed to discover the latent principal component or factorial axes that represent non-correlated effects. PCA is a statistical procedure that uses an orthogonal transformation to convert a set of possibly correlated variables into a set of values of linearly uncorrelated variables called principal components. Normalized PCA has been selected to account for different scales of available KPIs and graphical results can be seen at Figure 2 on the right. Almost a $90 \%$ of the total variance in the variables is explained by the two first principal component 
variables. The first axis accounts for $60 \%$ of the total variance and divides KPIs into two subsets: throughput rate and speed oppose to demand and density KPIs, meaning that increasing total demand does mean decreasing the percentage of completed trips (throughput decreases) and also implies decreasing mean speed (due to more vehicles in the network and thus congestion effects). Total travel time (ttt.h) and demand (total vehicles) vectors in Figure 2 on the right show a low angle meaning a high correlation between them; that it is meaningful in terms of transport analysis.
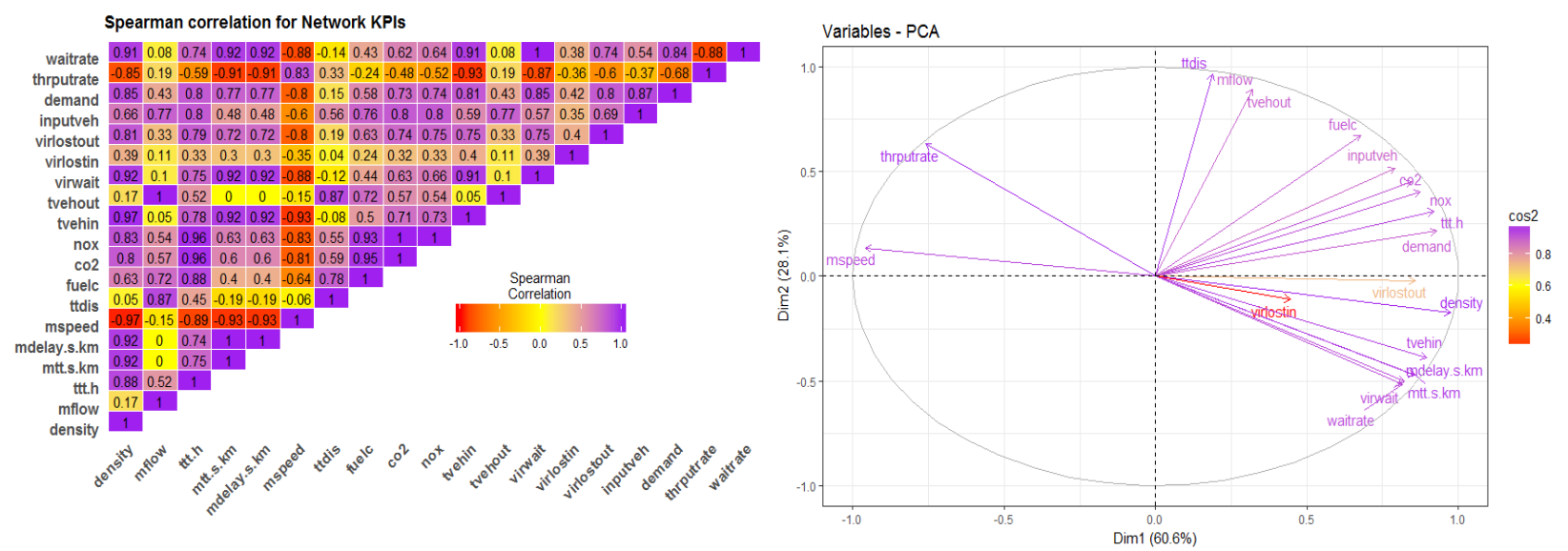

Figure 2. Assessment of correlation between System KPIs obtained by simulation using PCA.

On the second axis and orthogonal to the first one (see Figure 2 on the right), total travel distance (ttdis) and total completed trips (tvehout) show a high correlation between them, but total completed trips vector is almost orthogonal to virwait variable, total vehicles waiting to enter to the network; this last variable (virwait) is very correlated to mtt.s.km, density and delay.s.km that clearly reflect congestion level leading to the conclusion that despite congestion by increasing total travel distance, the number of completed trips seems to be not affected. Navigation strategies seem to play a role of increasing total travel distance in order to increase the number of completed trips (tvehout).

Congestion conditions are represented in the first factorial axis, positive values to the right (Figure 2 on the right) indicate high congestion and negative values represent higher speeds and lighter congestion. At the end of this section, we focus on delay while covering a km (delay.s.km) KPI, since it is a sensitive indicator of congestion, intuitive and with a high quality representation under the first factorial axis.

Figure 3 analyzes OD routes for an specific OD pair in a simulation run for a lane-based experiment. Selected routes by guided and non-guided drivers are shown and also route mean travel times, distances and comparison to global OD statistics (considering all vehicle types). Mean OD travel times for guided vehicles can be seen lower for non-guided vehicles, but mean OD travel distance for guided vehicle are greater than those for non-guided ones. This plot depicts which is commonly known by urban drivers: shortest distance routes are not the most convenient in terms of travel time to reach a destination. Consistency of selected OD routes for different OD pairs has been easily checked using CitScale analysis framework which simplifies the validation task.

Figure 4 shows some KPI results for a subset of experiments defined by the base-level composition of driver population (Expert-Regular-Tourist), a 20\% guidance penetration (GP), a demand level that assumes a $20 \%$ increment of the historic demand - as a consequence, the knowledge of recurrent congestion by expert drivers makes them to take non-optimal routes once historical conditions are altered. Time-window (TW factor) for data collection and elaboration of travel time estimates is fixed to $3 \mathrm{~min}$. Factors PVD and NS take different levels (non-exhaustive) and relative values for density, flow, travel time per $\mathrm{km}$, delay per $\mathrm{km}$, speed, total travel time, fuel consumption and $\mathrm{CO} 2$ and $\mathrm{NOx}$ emissions are calculated taken as a reference a link-based navigation and 0\% GP level. Clearly, density reduction is 
greater in lane-based scenarios than link-based ones. The same conclusion holds for travel time per km and delay per km (both in seconds). Speed increment is greater in lane-based scenarios than link-based.

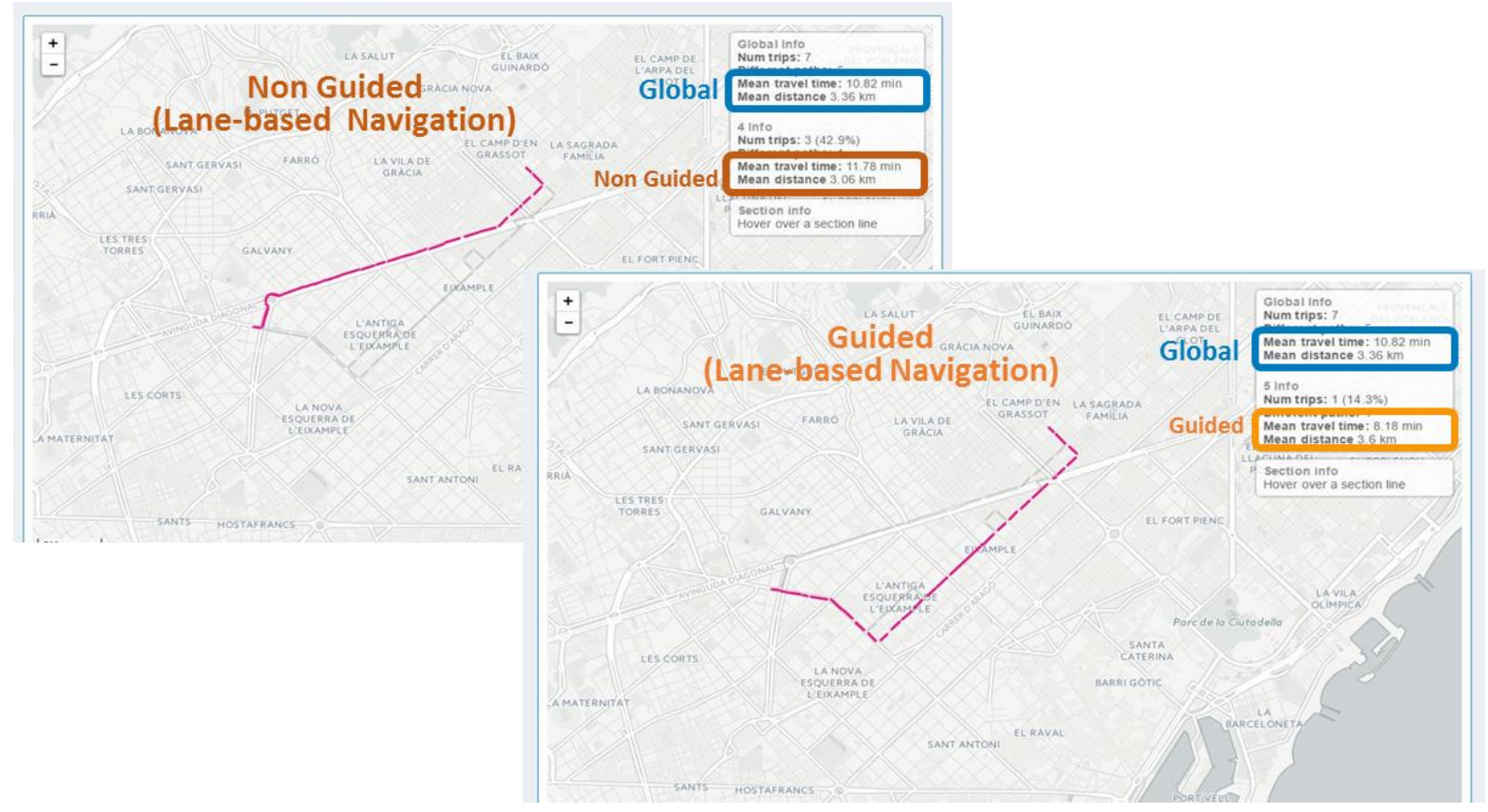

Figure 3. Visualization of alternative path according to driver-type for a selected od-pair.

\begin{tabular}{|c|c|c|c|c|c|c|c|c|c|c|c|c|c|c|}
\hline Drivers & $\begin{array}{l}\text { Navigation } \\
\text { Penetration }\end{array}$ & $\begin{array}{l}\text { Demand } \\
\text { Pattern }\end{array}$ & $\begin{array}{c}\text { Connected } \\
\text { Car } \\
\text { Penetration }\end{array}$ & $\begin{array}{c}\text { Time } \\
\text { Window }\end{array}$ & $\begin{array}{l}\text { Navigation } \\
\text { Strategy }\end{array}$ & density & mflow & ttt.h & mdelay.s.km & mspeed & ttdis & fuelc & $\mathrm{CO} 2$ & NOx \\
\hline $40-50-10$ & 20 & 20 & 0 & 3 & Link Navigation & $0,00 \%$ & $0,00 \%$ & $0,00 \%$ & $0,00 \%$ & $0,00 \%$ & $0,00 \%$ & $0,00 \%$ & $0,00 \%$ & $0,00 \%$ \\
\hline $40-50-10$ & 20 & 20 & 10 & 3 & Link Navigation & $-4,53 \%$ & $1,67 \%$ & $-5,48 \%$ & $-7,89 \%$ & $1,17 \%$ & $3,14 \%$ & $1,47 \%$ & $0,41 \%$ & $0,11 \%$ \\
\hline $40-50-10$ & 20 & 20 & 20 & 3 & Link Navigation & $-6,64 \%$ & $3,07 \%$ & $-6,33 \%$ & $-9,12 \%$ & $1,42 \%$ & $4,75 \%$ & $2,51 \%$ & $0,44 \%$ & $0,17 \%$ \\
\hline $40-50-10$ & 20 & 20 & 30 & 3 & Link Navigation & $-5,66 \%$ & $1,94 \%$ & $-7,28 \%$ & $-10,47 \%$ & $2,53 \%$ & $3,63 \%$ & $0,80 \%$ & $-0,26 \%$ & $-0,43 \%$ \\
\hline $40-50-10$ & 20 & 20 & 100 & 3 & Link Navigation & $-9,15 \%$ & $3,30 \%$ & $-9,12 \%$ & $-13,17 \%$ & $3,32 \%$ & $4,96 \%$ & $1,08 \%$ & $-0,95 \%$ & $-1,45 \%$ \\
\hline $40-50-10$ & 20 & 20 & Perfect Info & 3 & Link Navigation & $-8,93 \%$ & $3,91 \%$ & $-8,08 \%$ & $-11,72 \%$ & $2,62 \%$ & $5,37 \%$ & $1,94 \%$ & $-0,48 \%$ & $-1,04 \%$ \\
\hline $40-50-10$ & 20 & 20 & 0 & 3 & Lane Navigation & $-5,08 \%$ & $0,96 \%$ & $-4,01 \%$ & $-5,80 \%$ & $1,74 \%$ & $1,23 \%$ & $-0,98 \%$ & $-1,70 \%$ & $-1,86 \%$ \\
\hline $40-50-10$ & 20 & 20 & 10 & 3 & Lane Navigation & $-8,14 \%$ & $3,30 \%$ & $-8,34 \%$ & $-12,07 \%$ & $2,75 \%$ & $5,69 \%$ & $2,22 \%$ & $0,10 \%$ & $-0,12 \%$ \\
\hline $40-50-10$ & 20 & 20 & 20 & 3 & Lane Navigation & $-10,33 \%$ & $3,76 \%$ & $-9,67 \%$ & $-13,97 \%$ & $3,70 \%$ & $6,04 \%$ & $1,61 \%$ & $-0,89 \%$ & $-1,31 \%$ \\
\hline $40-50-10$ & 20 & 20 & 30 & 3 & Lane Navigation & $-9,80 \%$ & $2,48 \%$ & $-11,17 \%$ & $-16,13 \%$ & $4,63 \%$ & $4,43 \%$ & $-0,87 \%$ & $-2,04 \%$ & $-2,23 \%$ \\
\hline $40-50-10$ & 20 & 20 & 100 & 3 & Lane Navigation & $-12,85 \%$ & $4,63 \%$ & $-11,61 \%$ & $-16,79 \%$ & $4,90 \%$ & $6,77 \%$ & $1,25 \%$ & $-1,90 \%$ & $-2,45 \%$ \\
\hline $40-50-10$ & 20 & 20 & Perfect Info & 3 & Lane Navigation & $-9,95 \%$ & $3,14 \%$ & $-11,52 \%$ & $-16,63 \%$ & $4,56 \%$ & $5,05 \%$ & $0,09 \%$ & $-1,66 \%$ & $-1,94 \%$ \\
\hline
\end{tabular}

Figure 4. KPI figures for comparison to basic scenario (first row): lane vs link based navigation.

Dark cells in Figure 4 are remarkable since they refer to total travel distances (ttdis column)-showing an increment on total travel distance when PVD level increases, either in lane-based, or link-based scenarios that has to be properly assessed. Total travel distance is an absolute KPI and it depends on the number of vehicles entering the network during the simulation period (inputveh column) that was checked to increase, although not shown in Figure 4, leading to an increase of total travel distance, so this does not have to be interpreted as a drawback of increasing probe vehicle penetration. Emissions seem to slightly reduce as PVD penetration increases (improving the amount of data for travel time estimation), but reductions are not very important. Fuel consumption results are not conclusive, but a modest increment can be expected which can be justified attending to the increment of total travel distance that occurs when diverging to longer routes (in length) suggested by the navigation system. Rows linked to 0\% PVD are interesting, because navigation strategies in this case are fed with free-flow travel times leading 
inaccurate shortest paths (in time) for guided vehicles and show that lane-based navigation strategy outperforms results obtained for link-based navigation.

The linear model relating design factors to mean delay per $\mathrm{km}$ has a coefficient of determination of 97\% and all factors are statistically significant indicating the net-effect of all design factors. GP, PVD and DP are the most significant factors and TW shows lesser significance $\left(10^{-6}\right)$. According to Figure 5, as guidance penetration increases mean delay per $\mathrm{km}$ decreases up to $30 \%$, then it becomes stable and for a $100 \%$ guidance penetration slightly increases. Reduction in delay per $\mathrm{km}$ (s) from a non-guidance situation to a $30 \%$ guidance situation is remarkable $-35 \%$ reduction - this is an important result that indicates that guidance assistance is globally useful for the system and seems to benefit either guided, or non-guided vehicles. As PVD penetration increases, the quality of travel time estimates used to calculate reactive shortest paths for drivers that are navigation users seems to increase and the outcome in the global system is a significant decrease in mean delay per km that reaches a minimum when PVD is $90 \%$.

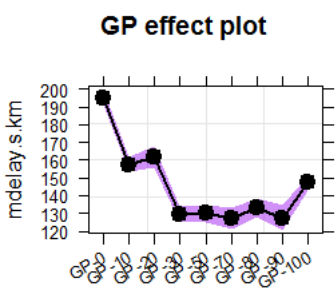

GP

NS effect plot

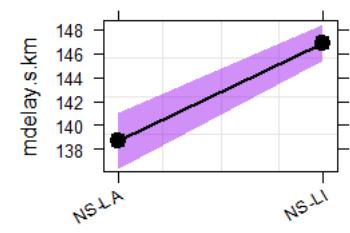

NS

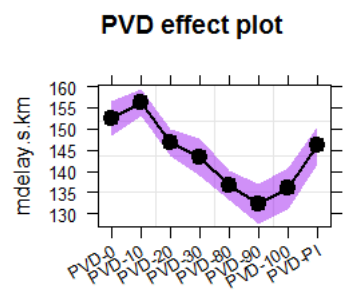

PVD

TD effect plot

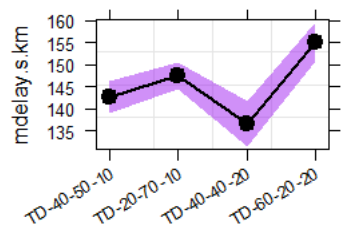

TD

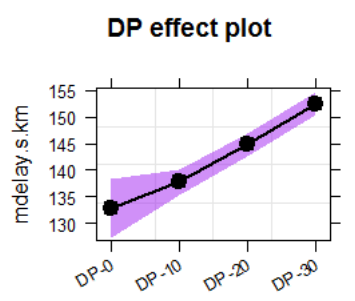

DP

TW effect plot

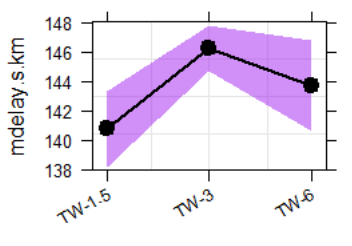

TW

Figure 5. Main effects on delay-time (seconds per $\mathrm{km}$ ) of experimental design factors: all experiments (95\% confidence bands).

Demand pattern factor is also very important, as one might expect, since it is defines the total number of trips wishing to travel over the network and common sense knowledge is confirmed with simulation results: as demand increases, mean delay per km increases as more congestion is present in the network. NS factor is the critical factor in this research, since new navigation strategies based on lane specific data are the aim of this paper and results confirm that lane-based navigation advise produces a reduction in mean delay per $\mathrm{km}$, thus perception of congestion is reduced and results point to a promising improvement of $7 \%$ reduction in delay. Speed, travel time and travel time to cover one $\mathrm{km}$ are alternative KPIs that have been analyzed and for all of them, a lane-based navigation advise indicates a benefit over traditional link-based navigation. A $30 \%$ GP seems to be the lower penetration rate to obtain global benefit for the whole population of drivers. According to PVD marginal plot in igure 5, a 10\% PVD penetration seems to be too low to get a global benefit in terms of congestion.

\section{CONCLUSIONS AND FUTURE RESEARCH}

In this paper, an approach consisting of a general framework and simulation architecture is used for emulating and evaluating general probe vehicle fleets and navigation strategies. The research carried out relies on a detailed simulation of the proposed driver classes and two reactive navigation strategies that have been revealed to be advantageous for guided and non-guided cars from $30 \%$ of guided vehicles. We 
conclude that any mobility service assessment must take into account several KPIs, since KPI are very correlated among them. Our simulation results confirm that lane-based navigation strategy represents an average benefit for the population of drivers, either guided, or non-guided, of 5-10\% the most affected KPIs (travel time, travel time per $\mathrm{km}$, delay per $\mathrm{km}$, speed). With respect to the benefit for specific driver classes analysis, in recurrent traffic conditions, navigation devices are not suitable for expert drivers, but they benefit from the use by other drivers. As a final remark that points to future research, data collected from probe vehicles has been proven to be useful for travel time estimates that are the base of shortest path routing provided by navigation strategies. Probe vehicle data is concluded by our simulation experiments to provide reliable travel time estimates to be included in dynamic OD matrix adjustment procedures as Kalman filtering schemes to simplify the space-estate model or SPSA formulations.

\section{ACKNOWLEDGMENTS}

This research was funded by TRA2016-76914-C3-1-P of the Spanish R+D National Programs and by the Secretaria d'Universitats i Recerca de la Generalitat de Catalunya under 2017 SGR 1749.

\section{REFERENCES}

Bessler, S., and T. Paulin. 2013. "Literature Study on the State of the Art of Probe Data Systems in Europe". Vienna: FTW Telecommunications Research Center.

Chiu, Y.-C., J. Bottom, M. Mahut, A. Paz, R. Balakrishna, T. Waller, and J. Hicks. 2011. "Dynamic Traffic Assignment: A primer". Transportation Research E-Circular (E-C153).

Deflorio, F. P. 2003. "Evaluation of a Reactive Dynamic Route Guidance Strategy". Transportation Research Part C: Emerging Technologies 11(5):375-388.

Fedorov, V. V. 1972. "Theory Of Optimal Experiments". In Probability and Mathematical Statistics. New York, Academic Press.

Hull, B., V. Bychkovsky, Y. Zhang, K. Chen, M. Goraczko, A. Miu, E. Shih, H. Balakrishnan, and S. Madden. 2006. "CarTel". In Proceedings of the 4th international conference on Embedded networked sensor systems - SenSys '06, November $1^{\text {st }}-3^{\text {rd }}$, Boulder, CO, USA, 125.

Lee, J., and B. Park. 2008. "Evaluation of Route Guidance Strategies Based on Vehicle-Infrastructure Integration Under Incident Conditions". Transportation Research Record: Journal of the Transportation Research Board 2086:107-114.

Lee, U., and M. Gerla. 2010. "A Survey of Urban Vehicular Sensing Platforms". Computer Networks 54(4):527-544.

Linares, M. P., L. Montero, E. Lorente, O. Serch, G. Navarro, J. Salmeron, and J. Casanovas. 2017. "Analytics Tool for Assessing Innovative Mobility Concepts, Vehicles and City Policies (CitScale)". In Proceedings of the 5th IEEE International Conference on Models and Technologies for Intelligent Transportation Systems, MT-ITS 2017, June $26^{\text {th }}-28^{\text {th }}$, Napoles, Italy, 385-390.

Lorkowski, S., P. Mieth, K.-U. Thiessenhusen, D. Chauhan, B. Passfeld, and R.-P. Schäfer. 2003. "Towards Area-Wide Traffic Monitoring-Applications Derived From Probe Vehicle Data". In Proceedings of the 8th International Conference on Applications of Advanced Technologies in Transportation Engineering, May $26^{\text {th }}-28^{\text {th }}$, Beijing, 1-6.

Minelli, S., P. Izadpanah, and S. Razavi. 2015. "Evaluation of Connected Vehicle Impact on Mobility and Mode Choice". Journal of Traffic and Transportation Engineering (English Edition) 2(5):301-312.

Montero, L., M. P. Linares, O. Serch, and J. Casanovas-Garcia. 2017. "A Visualization Tool Based on Traffic Simulation for the Analysis and Evaluation of Smart City Policies, Innovative Vehicles and Mobility Concepts". In Proceedings of the 2017 Winter Simulation Conference, edited by W. K. V. Chan et al., 3196-3207. Las Vegas, Nevada: IEEE.

Montero, L., M. Pacheco, J. Barceló, S. Homoceanu, and J. Casanovas. 2016. "Case Study on Cooperative Car Data for Estimating Traffic States in an Urban Network". Transportation Research Record: Journal of the Transportation Research Board 2594:127-137. 
Oh, J.-S., and R. Jayakrishnan. 2002. "Emergence of Private Advanced Traveler Information System Providers and Their Effect on Traffic Network Performance". Transportation Research Record: Journal of the Transportation Research Board 1783:167-177.

Olia, A., H. Abdelgawad, B. Abdulhai, and S. N. Razavi. 2016. "Assessing the Potential Impacts of Connected Vehicles: Mobility, Environmental, and Safety Perspectives". Journal of Intelligent Transportation Systems: Technology, Planning, and Operations 20(3):229-243.

Paikari, E., L. Kattan, S. Tahmasseby, and B. H. Far. 2013. "Modeling and Simulation of Advisory Speed and Re-routing Strategies in Connected Vehicles Systems for Crash Risk and Travel Time Reduction". In Proceedings of the $26^{\text {th }}$ Canadian Conference on Electrical and Computer Engineering (CCECE), May $5^{\text {th }}-8^{\text {th }}$, Regina, SK, Canada, 1-4.

Pan, J., M. A. Khan, I. S. Popa, K. Zeitouni, and C. Borcea. 2012. "Proactive Vehicle Re-routing Strategies for Congestion Avoidance". In Proceedings of IEEE 8th International Conference on Distributed Computing in Sensor Systems, DCOSS 2012, May $16^{\text {th }}-18^{\text {th }}$, Hangzhou, China ,265272.

RStudio Project, R. "Shiny Web Application framework for R". Accessed May, 2015. https://www.rstudio.com/products/shiny/. https://www.rstudio.com/products/shiny/.

Sanaullah, I., M. Quddus, and M. Enoch. 2013. "Estimating Link Travel Time from Low Frequency GPS Data". In Proceedings of the 92nd Transportation Research Board Annual Conference. January $13^{\text {th }}$ $-17^{\text {th }}$, Washington DC. https://trid.trb.org/view.aspx?id=1242269.

Transport Simulation Systems. 2014. "AIMSUN Microscopic Simulator (v 8.0)". Accessed May, 2015. http://www.aimsun.com.

Wang, J., J. Lv, C. Wang, and Z. Zhang. 2017. "Dynamic Route Choice Prediction Model Based on Connected Vehicle Guidance Characteristics". Journal of Advanced Transportation 2017(1):1-8.

\section{AUTHOR BIOGRAPHIES}

LIDIA MONTERO obtained her PhD in Computer Science in 1993 at the Universitat Politècnica de Catalunya (UPC). She is Associate Professor at the Department of Statistics and Operations Research at the UPC. Her research concerns simulation-optimization issues, demand modeling, data analysis and models applied to transport. Email: lidia.montero@upc.edu (http://futur.upc.edu/LidiaMonteroMercade).

M. PAZ LINARES is a postdoc researcher at the inLab FIB (Barcelona Informatics School laboratory) in the area of Mathematical Programming, Logistics and Simulation. Her research concerns dynamic traffic assignment, traffic simulation and optimization. Email: mari.paz.linares@upc.edu.

JOSEP CASANOVAS-GARCIA is a full professor in Operations Research, specializing in Simulation systems. He is one of the founders of the Barcelona School of Informatics. He is also the director of inLab FIB, an active research lab transferring in technology to business. Email: josepk@fib.upc.edu.

GONZALO RECIO is a bachelor student of Computer Science at Universitat Politècnica de Catalunya (UPC). He is a junior researcher at inLab FIB in the area of Mathematical Programming, Logistics and Simulation. Email: gonzalo.recio@ fib.upc.edu.

ESTER LORENTE is a graduate in Computer Science at Universitat Politècnica de Catalunya (UPC) and currently enrolled in Master in Innovation and Research in Informatics. She is a junior researcher in the area of Mathematical Programming, Logistics and Simulation. Email: ester.lorente@upc.edu.

JUAN SALMERÓN is a graduate in Computer Science at Universitat Politècnica de Catalunya (UPC). Senior developer at inLab FIB in the area of Mathematical Programming, Logistics and Simulation. Email: juan.salmeron@fib.upc.edu. 